\title{
A 72-year-old male with worsening interstitial infiltrates and respiratory failure
}

\section{Case report}

On November 24, 2004, a 72-year-old male was admitted to the medical intensive care unit (ICU) with a history of worsening dyspnoea, chest pain and bilateral interstitial infiltrates. $\mathrm{He}$ was initially admitted 3 weeks earlier (November $4,2004)$ to the intensive coronary care unit (ICCU) due to a 10-day history of worsening exertion dyspnoea, pleuritic chest pain associated with diffuse changes of the ST-T segment on ECG and elevated cardiac troponin $\mathrm{T}$. While the admission chest radiograph was normal, echocardiography revealed mild pericardial effusion and the erythrocyte sedimentation rate was elevated. A tentative diagnosis of pericarditis was entertained and high-dose aspirin therapy ( $2 \mathrm{~g}$ per day) was initiated.

The patient's previous medical history was remarkable for diabetes mellitus type 2, hypertension and dyslipidaemia. He had a smoking history of 20 pack-years some 30 years previously. The combination of hypoxaemia (oxygen pressure $6.9 \mathrm{kPa}(52 \mathrm{mmHg})$ while breathing $100 \%$ oxygen by face mask), elevated D-dimer and a normal chest radiograph 1 day after admission led to a contrast-enhanced computed tomography (CT) scan being performed (figure 1), which excluded pulmonary emboli.

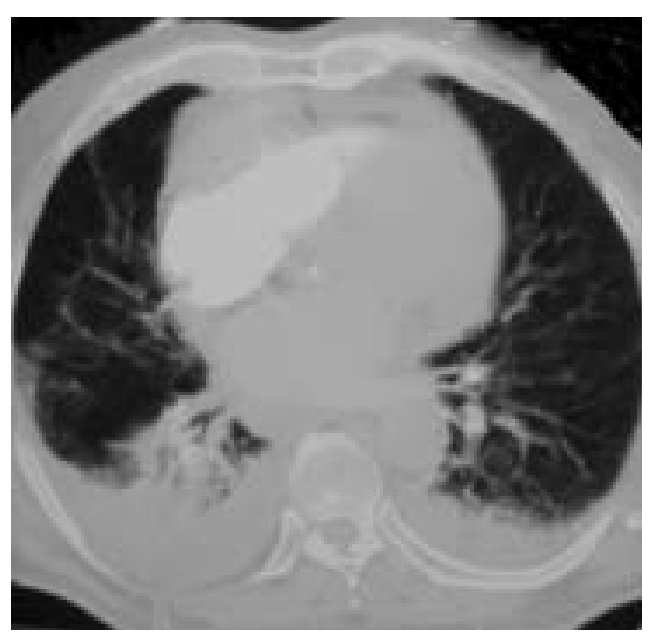

Figure 1

Contrast-enhanced CT scan of the chest.

\section{Task 1}

Interpret the pulmonary contrastenhanced CT.
V. Novack

L.S. Avnon ${ }^{2}$

N. Sion-Vardy ${ }^{3}$

Y. Almog

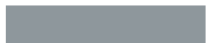

${ }^{1}$ The Medical Intensive Care Unit, ${ }^{2}$ Division of Pulmonology, and ${ }^{3}$ Dept of Pathology,

Soroka University Medical Center, Faculty of Health Sciences, BenGurion University,

Beer-Sheva, Israel.

\section{Correspondence:}

Y. Almog

MICU, Soroka University Medical

Center

POB 151

Beer-Sheva, 84101

Israel

Fax: 97286400166

E-mail:

almogya@bgumail.bgu.ac.il 


\section{Answer 1 \\ The contrast-enhanced CT scan of the chest showed small bilateral pleural effusion and pericardial effusion. There was no evidence of interstitial disease or mediastinal lymph nodes. On other sections, interstitial changes not seen on the admission chest radiograph were found in both apices.}

On the 2nd hospital day, rapid atrial fibrillation appeared, which was converted to sinus rhythm with intravenous amiodarone, and the patient was transferred to the medical floor on the 5 th hospital day. Amiodarone was administered for 8 days and the cumulative dose reached $5.2 \mathrm{~g}$ (loading dose of 1,050 $\mathrm{mg}$ followed by 7 days of $600 \mathrm{mg}$ per day). He remained on the floor for the next 2 weeks, where, despite therapy with aspirin, he continued to gradually deteriorate. The patient became progressively hypoxaemic with increasing oxygen requirements. Findings on repeat chest radiography, performed on the 18th hospital day (November 23, 2004), led to a repeat chest CT (figure 2).

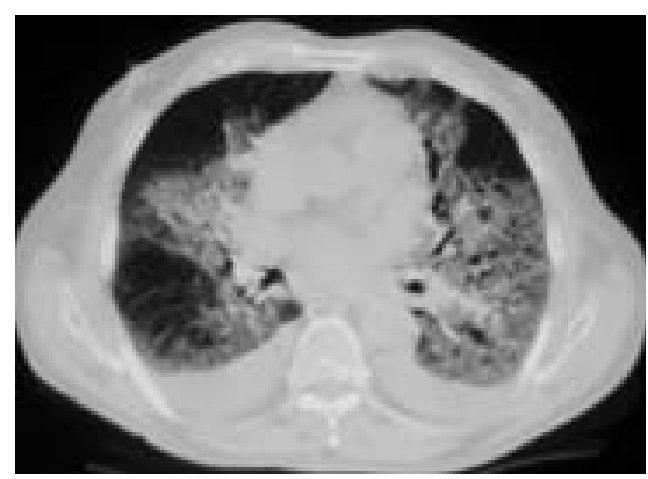

Figure 2

Second CT scan of the chest.

Task 2

Interpret the second chest CT.

\section{Answer 2}

The second CT scan of the chest showed a severe diffuse bilateral reticulo-interstitial pattern. Multiple small bullae were evident in both apices and in the left peri-hilar region. Bilateral pleural effusion was evident. There were no masses or mediastinal lymph nodes.

At this point the patient was transferred to the medical ICU. On admission, the patient was in severe respiratory distress, with 38 breaths per minute. While breathing $100 \%$ oxygen by face mask, oxygen saturation was $86 \%$. His temperature was $37^{\circ} \mathrm{C}$ and his heart rate was irregular, 110 beats per minute. Diffuse end-inspiratory crackles could be appreciated over the chest bilaterally. The rest of the physical examination was normal. The patient was intubated due to severe hypoxaemic respiratory failure requiring mechanical ventilation. Post-intubation chest radiography was performed (figure 3).

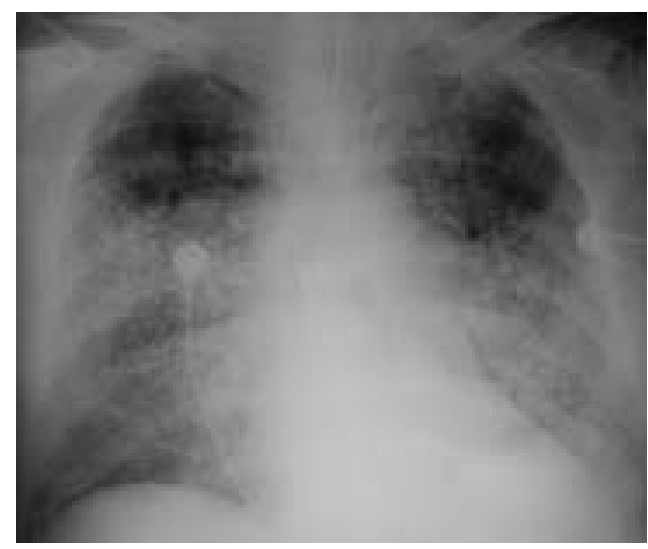

Figure 3

Chest radiography.

Task 3

Interpret the chest radiograph. 


\section{Answer 3}

Post-intubation chest film showed marked bilateral consolidation. The heart shadow was not enlarged.

Laboratory results showed a haemoglobin concentration of $10 \mathrm{~g} \cdot \mathrm{dL}^{-1}$, a creatinine level of 1.7 $\mathrm{mg} \cdot \mathrm{dL}^{-1}$ and a lactate dehydrogenase (LDH) level of $1,400 \mathrm{IU} \cdot \mathrm{L}^{-1}$. The C-reactive protein (CRP) level was $7.7 \mathrm{~g} \cdot \mathrm{dL}^{-1}$, and rheumatoid factor (RF), antinuclear antibody (ANA), perinuclear-antineutrophil cytoplasmic antibodies ( $p$-ANCA) and anti-glomerular basement membranes (antiGBM), as well as complement levels (C3 and C4), were all within the normal range. Acid-fast staining was negative.

At this stage, therapy with intravenous methylprednisolone $2 \mathrm{mg} \mathrm{kg}^{-1}$ was initiated. The combination of rapidly progressive respiratory failure with no evidence of systemic, malignant or infectious aetiology led to a transbronchial biopsy, which was performed on the 23rd hospital day (3rd medical ICU day).

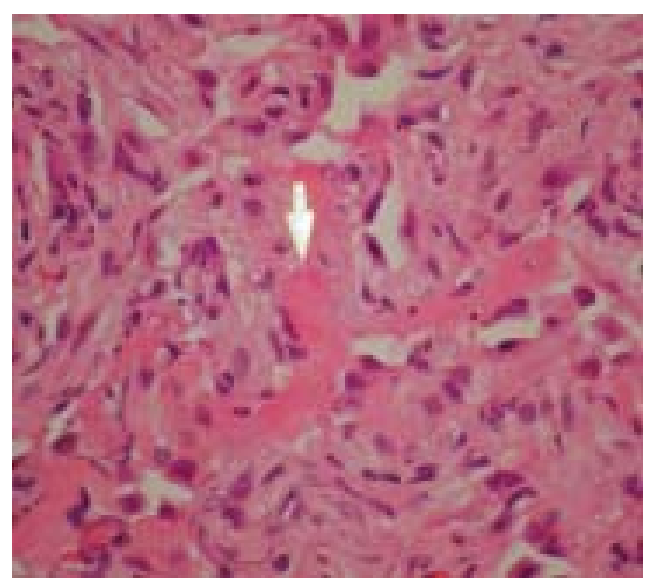

Figure 4

Transbronchial lung biopsy specimen.

\section{Answer 4}

The transbronchial lung biopsy specimen demonstrated late-stage diffuse alveolar damage (DAD) (organising DAD). There was evidence of organisation of hyaline membranes (arrow with marked interstitial fibroblast proliferation with residual alveolar spaces reduced to narrow slits). Regenerating alveolar epithelium was growing underneath the hyaline membranes. There were no granulomas or evidence for malignant or infectious process.

Despite therapy with corticosteroids, the patient remained mechanically ventilated with severe hypoxaemia and P/F ratios of $<100$. He expired due to severe sepsis and multi-organ failure on the 29th hospital day.

\section{Task 5 \\ Based on the information presented here, suggest a diagnosis.}

\author{
Task 4 \\ Interpret the histopathological \\ results.
}




\section{Answer 5 \\ Acute interstitial pneumonia (AIP) or Hamman-Rich syndrome leading to rapidly progressive hypoxaemic respiratory failure.}

\section{Discussion}

The interstitial lung diseases (ILDs) comprise a heterogeneous group of conditions that involve, in varying proportions, the various components of the lung parenchyma: alveoli, alveolar epithelium, capillary endothelium and the spaces between these structures. The ILDs are non-malignant, non-infectious disorders with unknown mechanism. The classification of ILDs is complex, and several pathological and clinical classifications have been proposed over the years. HAMMAN and $\mathrm{RICH}$ [1] were the first to describe (in 1935 and 1944) four patients who died of a rapidly progressive lung disease characterised by diffuse interstitial pneumonia and fibrosis. Over the subsequent years, many names have been coined, and the terminology used to describe this clinical entity was rather inconsistent [2, 3]. In 1986, the term "acute interstitial pneumonia" (AIP) was first introduced to describe a similar idiopathic clinical and pathological entity characterised by a rapid onset of interstitial lung disease leading to respiratory failure [4].

Histologically, AIP is characterised by a varying degree of $D A D$, fibroblastic proliferation and interstitial fibrosis [5]. In many respects, AIP resembles acute respiratory distress syndrome (ARDS) and could probably be viewed as a subtype of ARDS. The exact mechanisms responsible for AIP are poorly understood. As in other acute inflammatory conditions, it is the balance between pro-inflammatory mediators (e.g. cytokines, chemokines, oxygen radicals and eicosanoids) and anti-inflammatory factors (e.g. interleukin- 1 receptor antagonists, interleukin-10 and prostaglandin $\mathrm{I}_{2}$ ) that determines the final outcome [6].

The clinical features and course of AIP also resemble ARDS. It is the clinical context in which these two entities appear that distinguishes them. While ARDS typically occurs in the context of severe infections, sepsis, aspirations and trauma, no identifiable predisposing condition can be identified in AIP. The onset is usually acute (1-3 weeks), with dyspnoea and cough, followed by rapid development of respiratory failure and the requirement for mechanical ventilation in the vast majority of patients. Typically, a viral-like prodrome precedes the onset of symptoms in most patients. Fever is present in almost half of the patients on presentation, but typically a search for bacterial and viral aetiology will be negative [7]. In fact, the original description by HAMMAN and $\mathrm{RICH}$ [1] was of healthy individuals who presented with dyspnoea, cough and fever 10-20 days after a "flu-like" syndrome.

There is no apparent sex predilection and the mean age of onset is 50 years. The radiographic and CT features of AIP are similar to those of ARDS. High-resolution CT is more sensitive than chest radiography [6]. The early phases are characterised by ground-glass attenuation, while in the later phases consolidation and distortion dominate. AIP belongs to IIP; however, a wide variety of conditions may present with similar clinical features [8]. Idiopathic pulmonary fibrosis/cryptogenic fibrosing alveolitis (IPF/CFA) is a chronic progressive interstitial lung disease of unknown cause that results in respiratory failure and death with an average survival of 2-3 years. Most cases show a histological pattern of usual interstitial pneumonia (UIP), although a substantial minority have fibrotic nonspecific interstitial pneumonia. Patients with IPF/CFA also may have acute and typically terminal exacerbations, in which a histological pattern of DAD is superimposed on chronic changes [9]. The combination of acute onset, hypoxaemia out of proportion to radiological findings, lack of identifiable cause and, later, characteristic radiographical and pathological findings should alert the clinician to this distinct clinical entity. A lung biopsy is often required for a definitive diagnosis when AIP is suspected. It may be of great value in excluding important diagnostic considerations, such as CFA, infections, neoplasms, sarcoidosis and others.

In the present patient, the combination of rapidly progressive interstitial pneumonia leading to respiratory failure with no evidence for systemic, malignant or infectious aetiology led to a presumptive diagnosis of AIP or Hamman-Rich syndrome. Acute amiodarone-induced lung injury was also considered. However, the fact that the patient's symptoms clearly started before amiodarone was ever administered, as well as the clinical course, makes this diagnostic consideration quite unlikely.

Diagnosis was confirmed by a transbronchial biopsy performed on the 23rd hospital day (3rd medical ICU day) showing regenerating alveolar epithelium (DAD) and extensive interstitial 
fibroblast proliferation. There were no granulomas or evidence for malignant or infectious process.

Treatment of AIP is largely supportive and consists of non-invasive or invasive ventilatory support. Most clinicians will try corticosteroids in doses ranging $2-4 \mathrm{mg} \cdot \mathrm{kg}^{-1}$, even though there are no data to support this practice. Effectiveness of steroids is probably dependent upon early diagnosis and the extent and ratio of inflammation and fibrosis at the time of diagnosis. Some authors advocate the addition of immunosuppressive therapy in the form of cyclophosphamide or vincristine. Given the rapid course of AIP and the extent of fibrosis typically evident in biopsies, these agents are unlikely to affect the natural course, especially when fibrosis is extensive. In those patients who present with a more indolent clinical course and fail to respond to corticosteroids, lung transplantation should also be considered. Prognosis is poor and the mean 6month mortality of patients with AIP is $80 \%$ [7].

\section{Editorial comment}

Acute interstitial pneumonia has the worst prognosis among the seven disease entities of idiopathic interstitial pneumonia. Mortality rates exceed $70 \%$ in 3 months, despite mechanical ventilation [1]. It is frequently confused with other clinical entities characterised by rapidly progressive interstitial pneumonia, especially secondary acute interstitial pneumonia, acute exacerbations and accelerated forms of cryptogenic fibrosing alveolitis [2]. Furthermore, many authors use the above terms both erroneously and interchangeably. To differentiate between these entities, a large sample of lung tissue is needed. Video-assisted thoracoscopic biopsy is preferred [3]. Clinicians and especially intensivists should be aware of this rarely reported disease entity, in part due to its inclusion in ARDS cases.

References

1. Bouros D, Nicholson AC, Polychronopoulos V, du Bois RM. Acute interstitial pneumonia. Eur Respir J 2000; 15: 412-418.

2. Rice $A J$, Wells $A U$, Bouros $D$, et al. Terminal diffuse alveolar damage in relation to interstitial pneumonias. An autopsy study. Am J Clin Pathol 2003; 119: 709-714.

3. Case records 40-2002. A 56-year-old man with rapidly worsening dyspnea. N Engl J Med 2002; 347: 2149-2157.

\section{References}

1. Hamman L, Rich A. Acute diffuse interstitial fibrosis of the lung. Bull Johns Hopkins Hosp 1944; 74: 177-212.

2. Nicholson AG. Classification of idiopathic interstitial pneumonias: making sense of the alphabet soup. Histopathology 2002; 41: 381-391.

3. Poletti V, Kitaichi M. Facts and controversies in the classification of idiopathic interstitial pneumonias. Sarcoidosis Vasc Diffuse Lung Dis 2000; 17: 229-238.

4. Katzenstein A-LA, Myers JL, Mazur MT. Acute interstitial pneumonia: a clinicopathologic ultrastructural and cell kinetic study. Am J Surg Pathol 1986; 10: 256-267.

5. Katzenstein ALA, Myers JL. Idiopathic pulmonary fibrosis clinical relevance of pathologic classification. Am J Respir Crit Care Med 1998; 157: 1301-1315.

6. Bouros D, Nicholson AC, Polychronopoulos V, du Bois RM. Acute interstitial pneumonia. Eur Respir J 2000; 15: 412-408.

7. Bonaccorsi A, Cancellieri A, Chilosi M, et al. Acute interstitial pneumonia: report of a series. Eur Respir J 2003; 21: 187-191.

8. Travis WD, King TE, Bateman ED, et al. ATS/ERS international multidisciplinary consensus classification of idiopathic interstitial pneumonias. General principles and recommendations. Am J Respir Crit Care Med 2002; 165: 277-304.

9. Rice AJ, Wells $A U$, Bouros D, et al. Terminal diffuse alveolar damage in relation to interstitial pneumonias. An autospy study. Am J Clin Pathol 2003; 119: 709-714. 